\title{
Needs Assessment and System Design of an Extracorporeal Membrane Oxygenation Simulation Learning System
}

\author{
Kai-Ling CHI, RN, MS student ${ }^{\mathrm{a}, 1}$ and Shwu-Fen CHIOU, RN, $\mathrm{PhD}^{\mathrm{b}}$ \\ ${ }^{a}$ Nursing Department, Taipei Veterans General Hospital, Taiwan, ROC \\ ${ }^{\mathrm{b}}$ Department of Nursing, National Taipei University of \\ Nursing and Health Sciences, Taiwan, ROC
}

\begin{abstract}
Nurses require training to manage extracorporeal membrane oxygenation (ECMO). Thus, the aim of this study is to investigate intensive care unit nurse needs and design an ECMO simulation learning system. A systemic needs analysis included interviews and a literature review. Nurses had three learning needs: preECMO placement care, daily care during placement, and care for preparation of removal. The system design includes ECMO care knowledge, practice in simulations, and self-examination with a flexible learning methodology.
\end{abstract}

Keywords. Extracorporeal membrane oxygenation, simulation learning system, need assessment, system design

\section{Introduction}

With advancements in medical techniques and technologies, extracorporeal membrane oxygenation (ECMO) is increasingly being used to gain time for future treatment of many immediately lethal diseases. Therefore, ICU nurses must be able to manage this complex and sophisticated device to provide emergency treatment. In Taiwan, current ECMO educational training is mainly thru lectures and verbal instructions with a lack of diversified learning approaches. Today, digital simulation learning systems offer virtual learning situations and are able to provide instant and flexible learning opportunities.[1] As such, the aim of this study is to analyze the learning needs of ICU nurses in terms of an ECMO simulation learning system, and to develop a simulation learning system prototype according to such requirements.

\section{Methods}

This study was conducted according to the analysis and design stages of system development. This study used interviews and literature review to analyze the needs for the system. We invited five nurses from the ICU of the Department of Internal Medicine and Department of Surgery in a medical center in northern Taiwan for interviews. After

${ }^{1}$ Corresponding Author, Kai-Ling Chi, RN, ICU, Nursing Department, Taipei Veterans General Hospital, No. 201, Sec. 2, Shipai Road, Beitou District, Taipei City, Taiwan 11217, ROC; E-mail: kylin023@gmail.com. 
summarizing the interview data and literature reports, we designed a system prototype and planned the system framework, system content and function, and system interface. Subsequently, we invited five ICU nurses, clinical care experts, education experts, and others to provide comments on the system design content as the basis for system development. Upon completion of the system prototype development, this study utilized the "System Usability Scale (SUS)" and "Questionnaire for User Interface Satisfaction (QUIS)" to develop the research questionnaire and used convenience sampling to select ICU nurses to assess system usability and provide comments.

\section{Results}

The preliminary results are as follows: (1) System needs assessment: The system content should include pre-ECMO placement care, daily care during ECMO placement, and care for preparation of ECMO removal. Pre-ECMO placement care focuses on the preparation of materials required for placement of ECMO and assistance in placement care. Daily care during ECMO placement includes monitoring items, interpretation of examination reports, catheter care, function setting, and handling of complications and abnormal situations. Care for the preparation of removal includes the conditions for removal of ECMO and associated care.

(2) System prototype design: The system prototype design includes system user types, system content, system functions, and development tools. The users include nurses, teachers, and system managers. The system content includes ECMO care knowledge, simulation situations, self-examination, etc. The system functions include a search and browsing function of learning content, performing of ECMO care in simulation situations, test question design and testing, recording and search of learning history, and account management. The development tools included Dreamweaver CS6 to design webpages, PHP and MySQL to design the database, and Python and Scratch software to design interactive animations and simulation practice sessions.

\section{Conclusions}

This study reviewed the relevant literature regarding content of an ECMO simulation learning system from the perspective of the learning needs of ICU nurses. A system prototype was developed according to the results of the learning needs analysis. The system developed provides traditional teaching content and a variety of simulation situations of ECMO care. These simulations enable nurses to learn thru simulation practice, which reduces their anxiety in actual future care. This system offers ECMO care knowledge and simulations to practice appropriate skills thru repeated and flexible learning. As such, it may become a practical learning tool and effective methodology for in-service education.

\section{References}

[1] Hudgins K. Clinical simulation learning in critical care. Critical Care Nursing Quarterly, 40 (2017), 108110 . 